\title{
Significant photoinduced refractive index change observed in porous silicon Fabry-Pérot resonators
}

\author{
Morio Takahashi ${ }^{\mathrm{a})}$ and Yuichi Toriumi \\ Department of Electrical and Electronic Engineering, Faculty of Technology, Tokyo University \\ of Agriculture and Technology, Koganei, Tokyo 184-8588, Japan \\ Takahiro Matsumoto and Yasuaki Masumoto \\ Single Quantum Dot Project, ERATO-JST, 5-9-9 Tokodai, Tsukuba 300-2635, Japan \\ Nobuyoshi Koshida \\ Department of Electrical and Electronic Engineering, Faculty of Technology, Tokyo University \\ of Agriculture and Technology, Koganei, Tokyo 184-8588, Japan
}

(Received 27 July 1999; accepted for publication 14 February 2000)

\begin{abstract}
It is shown that a porous silicon Fabry-Pérot resonator device exhibits a significant nonlinear optical transmission behavior at wavelengths in the near-infrared region. The input-output (transmission-incident power) response curve shows superlinear or sublinear behavior dependent on excitation wavelengths around the resonant position. These characteristics are interpreted as a result of nonlinear refractive index change, including by enhanced optical power in the Fabry-Pérot cavity configuration and carrier accumulation in silicon nanocrystallites. The result leads to further progress of nanocrystalline silicon into functional photonic device applications. (c) 2000 American Institute of Physics. [S0003-6951(00)03815-8]
\end{abstract}

Electro-optical devices such as self-electro-optic effect devices $^{1}$ or all-optical devices such as optical bistable Fabry-Pérot étalons ${ }^{2,3}$ have been demonstrated based on the excitonic absorption effect in the quantum wells of III-V semiconductors. Large-scale integration of these photonic devices, however, has not been established because of the difficulty of monolithic processing.

In recent years, optical nonlinearities in nanoscale silicon crystallite materials including porous silicon (PS) have been reported. ${ }^{4-10}$ They show a significantly large photoinduced absorption effect. Especially, PS seems to be attractive for monolithic optoelectronic integration, since it emits visible electroluminescence efficiently. ${ }^{11,12}$

From a viewpoint of fabricating Fabry-Pérot resonators, it is an advantageous feature of PS that highly reflective multilayer mirrors can be obtained simply by controlling the anodization current density and time. ${ }^{13-15}$ Thus, PS offers the possibility of integrating optoelectronic devices on a $\mathrm{Si}$ substrate.

In this letter, we report the optical nonlinear effect induced by a refractive index change in a PS-based FabryPérot resonator (PS-FPR). Theoretical analyses suggest that the nonlinear refractive index change originates from carrier accumulation in silicon nanocrystallites.

Figure 1 shows a cross-sectional observation of a fabricated PS-FPR on a silicon substrate by a scanning electron microscope (SEM). The resonator, composed of an active layer and two distributed Bragg reflectors (DBRs), ${ }^{13-15}$ was prepared by anodizing $p$-type (100) Si wafers (0.1-0.5 $\Omega \mathrm{cm}$ ) in a mixture of $55 \mathrm{wt} \% \mathrm{HF}$ :ethanol $=1: 1$ at $0{ }^{\circ} \mathrm{C}$. To fabricate the DBRs, the anodization current density was modulated between 20 and $250 \mathrm{~mA} / \mathrm{cm}^{2}$ for periods of 5.47

${ }^{a)}$ Electronic mail: morio@cc.tuat.ac.jp and $1.06 \mathrm{~s}$, respectively, in order to obtain the same optical thickness as a quarter of the resonant wavelength with different refractive indices. The corresponding refractive indices are 2.60 and 1.54 , respectively. To fabricate the active layer, of which the optical thickness is equal to a resonant wavelength, the anodization current density and time are adjusted to $20 \mathrm{~mA} / \mathrm{cm}^{2}$ and $21.9 \mathrm{~s}$, respectively. The fabricated PS-FPR was peeled out from the silicon substrate electrochemically and was bonded on a quartz glass for the transmission measurement.

Figure 2(a) shows the plots of a transmission spectrum obtained by a spectrophotometer. The resonant wavelength is located at $807 \mathrm{~nm}$ as designed. The finesse $\mathcal{F}$ defined as $\pi \sqrt{R} /(1-R)$ is 19.3 in the present PS-FPR, where $R$ is the reflectivity of the DBRs. Nonlinear transmission experiments were performed using this PS-FPR and a continuous-wave $\mathrm{Ti}: \mathrm{Al}_{2} \mathrm{O}_{3}$ laser. These excitation wavelengths were selected at the resonant point $(808 \mathrm{~nm})$, the blue side $(800 \mathrm{~nm})$, and the red side $(816 \mathrm{~nm})$, as shown by the arrows of Fig. 2(a). The incident laser beam was modulated with a frequency of

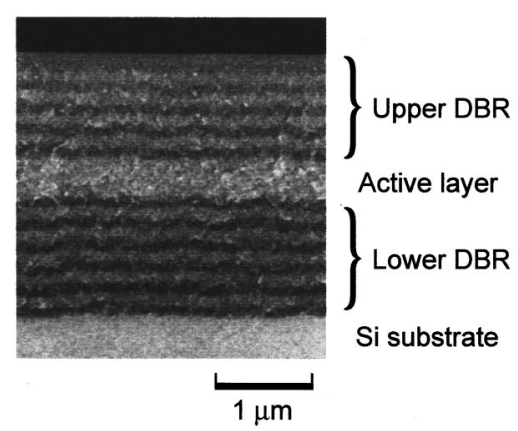

FIG. 1. SEM cross-sectional view of the PS-FPR with a resonant wavelength of $807 \mathrm{~nm}$. The DBRs were fabricated on both sides of the active PS layer. 

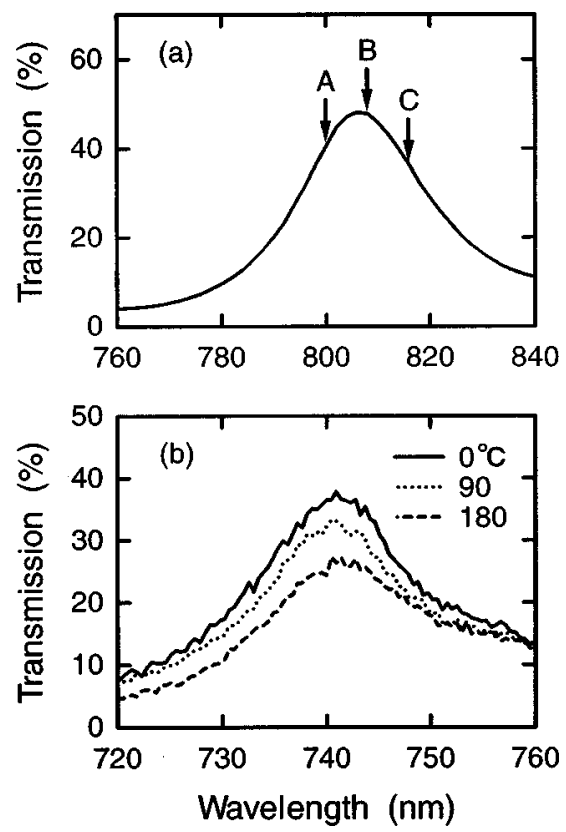

FIG. 2. (a) Linear transmission spectrum of the measured PS-FPR. The indicated points are excitation wavelengths as (A) $800 \mathrm{~nm}$, (B) $808 \mathrm{~nm}$, and (C) $816 \mathrm{~nm}$. (b) Temperature dependence of the transmission spectrum in the range from 0 to $180^{\circ} \mathrm{C}$.

$100 \mathrm{~Hz}$ using an acousto-optic modulator. The maximum optical power of the input wave form is $480 \mathrm{~W} / \mathrm{cm}^{2}$.

There might be thermal effects on the experimental results under the above-mentioned intense excitation conditions. So, prior to the nonlinearity measurement, the transmission spectra were measured separately at different temperatures in the range from 0 to $180^{\circ} \mathrm{C}$, as shown in Fig. 2(b). It is clear that the resonant wavelength remains at 740 $\mathrm{nm}$, although the transmission is slightly decreased in the whole wavelength range, possibly by thermally induced absorption. The implication is that the refractive index remains about the same in this temperature range.

When the laser beam was introduced into the PS-FPR, the transmission intensity (output) was measured as a function of the incident intensity (input) using silicon photodetectors and a digital oscilloscope. Figure 3 shows the measured wave forms and the corresponding input-output curves. Dependent on the excitation wavelength, the inputoutput curve shows different types of hysteretic loops. For excitation at $800 \mathrm{~nm}$, the transmission decreases sublinearly, as shown in Figs. 3(A) and 3( $\left.\mathrm{A}^{\prime}\right)$. At $816 \mathrm{~nm}$, in contrast, the transmission increases superlinearly, as shown in Figs. 3(C) and $3\left(\mathrm{C}^{\prime}\right)$ with increasing the incident optical power. We can exclude the induced absorption effects (absorption change), because the input-output curve at the resonant position is almost linear, as shown in Figs. 3(B) and 3(B'), even in higher excitation intensities. Furthermore, the superlinear behavior is inconsistent with the simple photoinduced or thermally induced absorption model in which only the sublinear should appear, as expected from Fig. 2(b). The distinct hysteretic transmission curves which are sensitive to the excitation wavelength strongly suggest that these nonlinearities originate from a refractive index change due to enhanced photon density in the cavity.

To understand the nonlinear behavior in the PS-FPR, we Downloaded 23 Jan 2008 to 130.158.56.123. Redistribution subject to AIP license or copyright; see http://apl.aip.org/apl/copyright.jsp

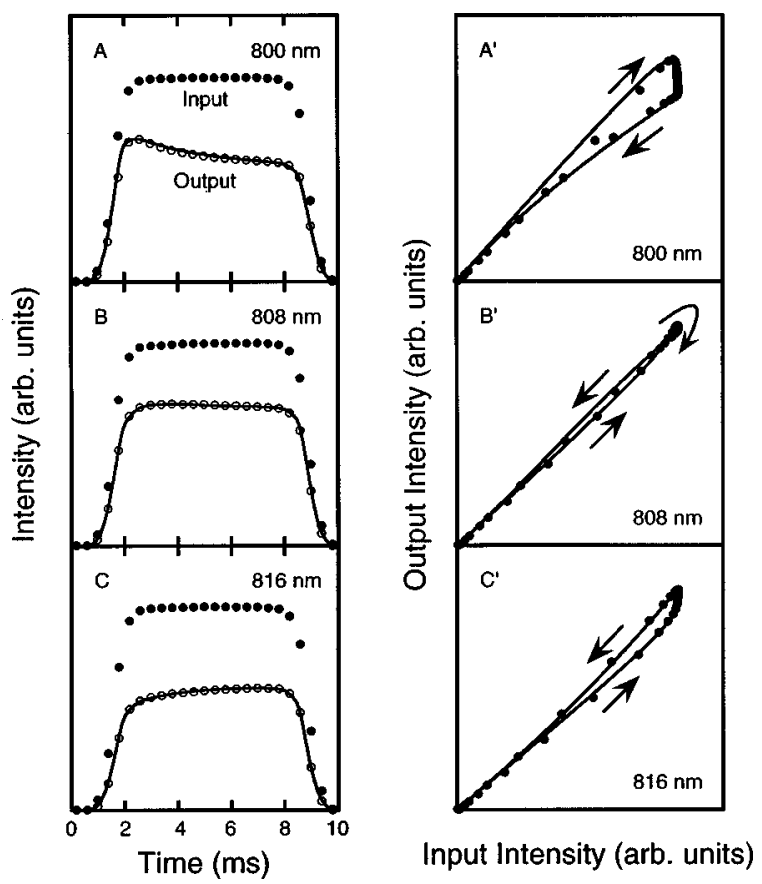

FIG. 3. Input (closed plots) and output (open plots) intensity wave forms and fitted curves (solid line) at various excitation wavelengths which correspond to the three points (A), (B), and (C) indicated in Fig. 2. The corresponding input-output hysteresis loops $\left(\mathrm{A}^{\prime}\right),\left(\mathrm{B}^{\prime}\right)$, and $\left(\mathrm{C}^{\prime}\right)$ are also shown by the closed plots (experimental) and the solid curves (fitted).

assume that a nonlinear refractive index change is caused by carrier accumulation in the active layer. Under this assumption, the nonlinear PS-FPR transmission intensity as a function of photoinduced refractive index change is given by

$$
I_{T}=\frac{T^{2} e^{-\alpha l}}{\left(1-\operatorname{Re}^{-\alpha l}\right)^{2}+4 \operatorname{Re}^{-\alpha l} \sin ^{2}\left\{\frac{2 \pi l}{\lambda}\left(n+n_{\mathrm{NL}} I_{T}\right)\right\}} I_{i},
$$

where $I_{T}$ is the resonator transmission intensity; $I_{i}$ is the intensity of the incident laser beam; $T$ is the transmission of DBRs; $\alpha, l$, and $n$ are the absorption coefficient, the length, and the linear refractive index of the active layer; $\lambda$ is the measured wavelength; the product of $n_{\mathrm{NL}} I_{T}$ is the photoinduced nonlinear refractive index change; and $n_{\mathrm{NL}}$ is the nonlinear coefficient of the change in refractive index. The transmission curve of Fig. 2(a) can be well fitted by Eq. (1) using values of $I_{i}=1, T=15 \%, R=85 \%, \alpha l=0.07, n l=807 \mathrm{~nm}$ and $n_{\mathrm{NL}}=0$ (linear response).

To explain sublinear and superlinear transmission curves obtained in Fig. 3, we consider two-level states as a relaxation path of carriers in PS-the strong incident optical power excites a large number of carriers to the conduction band, and then the excited carriers are thermalized to the conduction-band edge, where the thermalized carriers are accumulated due to its slow decay rate. The accumulated carriers are possible to change $n_{\mathrm{NL}}$. In this case, $n_{\mathrm{NL}}$ is proportional to the number of accumulated carriers and can be expressed as

$$
n_{\mathrm{NL}}=\gamma^{\prime} e^{-\eta t} \int_{0}^{t} e^{-\eta t^{\prime}} I_{T}\left(t^{\prime}\right) d t^{\prime},
$$


where $\gamma^{\prime}$ is proportional to the thermalization decay rate, and $\eta$ is the carrier lifetime for relaxation from the conduction band to the valence band.

Based on Eqs. (1) and (2), the hysteretic input-output curves can be fitted by the measured input intensity. The fitted curves obtained by this calculation are shown in Fig. 3 by solid curves. Here, the fitting parameters are chosen as $\gamma^{\prime}=0.190, \eta=202$ at $800 \mathrm{~nm}, \gamma^{\prime}=0.263, \eta=517$ at 808 $\mathrm{nm}$, and $\gamma^{\prime}=0.522, \eta=766$ at $816 \mathrm{~nm}$. The response time derived from the $\eta$ value is several milliseconds, which is consistent with the slow phtoluminescence decay components of PS of the order from microsecond to millisecond. According to the fitting treatment of the transmission change in Fig. 3, the nonlinear coefficient $n_{\mathrm{NL}}$ estimated is about $10^{-5} \mathrm{~cm}^{2} / \mathrm{W}$ at $816 \mathrm{~nm}$ at $I_{i}=480 \mathrm{~W} / \mathrm{cm}^{2}$. This nonlinear coefficient is consistent with the previous reports $\left(10^{-5}-10^{-7} \mathrm{~cm}^{2} / \mathrm{W}\right) .^{7,10}$ It is possible to obtain a much larger transmission change and fast response by improving the finesse of this cavity. The finesse may be improved by increasing the viscosity of the electrolyte by lowering the anodization temperature $\left(<0{ }^{\circ} \mathrm{C}\right)$ in order to fabricate the multilayer structure with fine interfaces. ${ }^{16}$

In conclusion, we have observed distinct sublinear and superlinear optical response curves in the PS-FPR. The nonlinear behavior in the PS-FPR can be well explained theoretically by a significant photoinduced change in the refractive index of the active nanocrystalline PS. The nonlinear refractive index change is presumably caused by carrier accumulation in silicon nanocrystallites. The nonlinear effect in the PS-FPR reported here shows promising aspects for monolithic photonic integration.
This work was partially supported by a Grant-in-Aid from the Ministry of Education, Science, Sports and Culture of Japan.

${ }^{1}$ D. A. B. Miller, D. S. Chemla, T. C. Damen, A. C. Gossard, W. Wiegmann, T. H. Wood, and C. A. Burrus, Appl. Phys. Lett. 45, 13 (1984).

${ }^{2}$ J. F. Heffernan, M. H. Moloney, J. Hegarty, J. S. Roberts, and M. Whitehead, Appl. Phys. Lett. 58, 2877 (1991).

${ }^{3}$ P. F. Davies, C. C. Philips, and C. Roberts, Appl. Phys. Lett. 74, 3717 (1999).

${ }^{4}$ T. Matsumoto, N. Hasegawa, T. Tamaki, K. Ueda, T. Futagi, H. Mimura, and Y. Kanemitsu, Jpn. J. Appl. Phys., Part 2 33, L35 (1994).

${ }^{5}$ T. Matsumoto, M. Daimon, H. Mimura, Y. Kanemitsu, and N. Koshida, J. Electrochem. Soc. 142, 3528 (1995).

${ }^{6} \mathrm{~T}$. Matsumoto and Y. Masumoto, Properties of Porous Silicon (INSPEC, London, 1997), Sec. 8.4, p. 238.

${ }^{7}$ F. Z. Henari, K. Morgenstern, W. J. Blau, V. A. Karavanskii, and V. S. Dneprovskii, Appl. Phys. Lett. 67, 323 (1995).

${ }^{8}$ P. Mary, J. Kudrna, F. Trojanek, and A. Hospodkova, Thin Solid Films 276, 84 (1996).

${ }^{9}$ V. Klimov, D. McBranch, and V. Karavanskii, Mater. Res. Soc. Symp. Proc. 405, 179 (1996)

${ }^{10}$ S. Vijayalakshmi, M. A. George, and H. Grebel, Appl. Phys. Lett. 70, 708 (1997).

${ }^{11}$ N. Koshida and H. Koyama, Appl. Phys. Lett. 60, 347 (1992).

${ }^{12}$ B. Gelloz, T. Nakagawa, and N. Koshida, Mater. Res. Soc. Symp. Proc. 536, 15 (1999).

${ }^{13}$ G. Vincent, Appl. Phys. Lett. 64, 2367 (1994).

${ }^{14}$ M. G. Berger, C. Dieker, M. Thönissen, L. Vescan, H. Lüth, H. Münder, W. Theiss, M. Wernke, and P. Grosse, J. Phys. D 27, 1333 (1994).

${ }^{15}$ M. Araki, H. Koyama, and N. Koshida, Jpn. J. Appl. Phys., Part 135 , 1041 (1996).

${ }^{16}$ S. Setzu, S. Létant, P. Solsona, R. Romestain, and J. C. Vial, J. Lumin. 80, 129 (1999). 